\title{
Autophagy induction on impaired spermatogenesis of xeroderma pigmento- sum group A gene-deficient mice
}

\author{
Hironobu Nakane ${ }^{1}$, Katsumi HigakI ${ }^{2}$, Yuka Koyama ${ }^{1}$, Eiji NANBA ${ }^{3}$, and Toshiyuki KaIDOH ${ }^{1}$ \\ ${ }^{1}$ Department of Anatomy, Faculty of Medicine, Tottori University, Nishi-cho 86, Yonago, Tottori 683-8503, Japan; ${ }^{2}$ Research Initiative \\ Center; and ${ }^{3}$ Research Strategy Division, Organization for Research Initiative and Promotion, Tottori University, Nishi-cho 86, Yonago, \\ Tottori 683-8503, Japan
}

(Received 3 April 2020; and accepted 30 July 2020)

\begin{abstract}
Xeroderma pigmentosum (XP) involves a defect in the initial step of nucleotide excision repair (NER) and consists of eight genetic complementation groups (groups $\mathrm{A}-\mathrm{G}$ and a variant). XP group A (XPA) patients have a high incidence of UV-induced skin tumors, immature testicular development, and neurological symptoms. In an earlier study, we have shown that XP group A (Xpa) gene-knockout mice $\left(\mathrm{Xpa}^{-/-}\right.$mice) were highly sensitive to UV-induced skin carcinogenesis with a defect in NER and were highly susceptibility to spontaneous tumorigenesis with impaired spermatogenesis. However, the pathology of impaired spermatogenesis in $\mathrm{Xpa}^{-/-}$mice is unknown. To unravel the underlying pathology, we made a concerted effort using the testis of 3-month-old $\mathrm{Xpa}^{-/-}$mice. We found many large vacuoles in the seminiferous tubules of 3-month old $\mathrm{Xpa}^{-/-}$ mice, while there were no large vacuoles in that of $\mathrm{Xpa}^{+/+}$mice. Immunohistochemistry of microtubule-associated protein 1 light chain 3 (LC3), an autophagosome marker, showed degenerating cells with intense signal of LC3 in the seminiferous tubules, and immunoblotting revealed induction of LC3-II in the 3-month-old $\mathrm{Xpa}^{-/-}$mice. The results of the present study suggest autophagy induction as the possible mechanism underlying the impaired spermatogenesis in $\mathrm{Xpa}^{-/-}$mice. Therefore, $\mathrm{Xpa}^{-/-}$mice could be a useful model for investigating aging and male infertility with low expression of $X P A$.
\end{abstract}

\section{INTRODUCTION}

Xeroderma pigmentosum (XP) is an autosomal recessive disease marked by an extreme hypersensitivity to sunlight, susceptibility to skin cancer in sun-exposed areas, and severe neurological abnormalities. Cells from XP patients show hypersensitivity to killing by UV-irradiation. In addition to these symptoms, XP group A patients have immature testicular development. There are eight genetic complementation groups in XP; XP group A through XP group $\mathrm{G}$

Address correspondence to: Dr Hironobu Nakane, Department of Anatomy, Faculty of Medicine, Tottori University, Nishi-cho 86, Yonago, Tottori 683-8503, Japan Tel: +81-859-38-6013, Fax: +81-859-38-6010

E-mail: mnakane@tottori-u.ac.jp and a variant (XPV). The primary defect in XP group A (XPA) through XP group G (XPG) ascribes in an early step of nucleotide excision repair (NER) whereas XPV has a normal NER process but a defect in translesion DNA synthesis. In the NER, XPA protein has a role in the verification of abnormalities in DNA chemistry with the helicase activity of transcription factor II $\mathrm{H}$ (TFIIH). To date, the genes responsible for NER and translesion synthesis associated with XP have been identified (XPA-XPG and $X P V$, respectively), and core NER reactions have been reconstituted using purified proteins including XPA-XPG proteins (DeSanctis and Cacchione 1932; Friedberg et al. 2006; Sugasawa 2016).

We have generated Xpa gene-knockout mice $\left(\mathrm{Xpa}^{-/-}\right.$ mice) by insertion of the neo gene into exon 4 of the mouse $X p a$ gene. The $X_{p a}^{-/-}$mice were found to 
be defective in NER and highly susceptible to ultraviolet-B(UVB)- or 9,10-dimethyl-1,2-benz[a]anthracene (DMBA)-induced skin carcinogenesis, therefore presenting as a suitable animal model to study the UVB-induced skin tumorigenesis in XP group A patients (Nakane et al. 1995). In another study using the $\mathrm{Xpa}^{-/-}$mice, we reported impairment of spermatogenesis with reduction of testis weight in an age-dependent manner and higher incidence of spontaneous tumorigenesis later in life. The relative weight ratio of the testis in the $\mathrm{Xpa}^{-/-}$mice was reduced to 87.5 percent of that in the $X p a^{+/+}$mice at 3 months old, half of that in the $X p a^{+/+}$mice at 12 months old and finally one-third of that in the $X_{p a}{ }^{+/+}$ mice at 24 months old. Male $\mathrm{Xpa}^{-/-}$mice were fertile until about 30 weeks of age. We found degenerating seminiferous tubules in the testes of $\mathrm{Xpa}^{-/}$ mice after 6 months of age. The degenerating seminiferous tubules were detected in a few percent of tubules at 6 months of $\mathrm{Xpa}^{-/-}$mice, half of tubules at 12 months of $\mathrm{Xpa}^{-/-}$mice, and all tubules at 24 months of $X_{p a} a^{-/}$mice. In contrast, there were no degenerating seminiferous tubules in the $\mathrm{Xpa}^{-/-}$mice at the age of 3 months (Nakane et al. 2008). We supposed that the endogenous DNA damages such as cyclopurine repaired by only NER might affect the relatively slow time course of impaired spermatogenesis in the $\mathrm{Xpa}^{-/-}$mice (Nakane et al. 2008; Brooks 2017). Recently, we detected a higher accumulation of oxidatively generated type of DNA damage called purine 8,5'-cyclo-2'-deoxynucleoside (cyclopurine) in their organs of 1-, 3-, 6-, 24-monthold $\mathrm{Xpa}^{-/}$mice than in those of wild type (wt) mice (Mori et al. 2019). Moreover, it has been reported that abnormalities in DNA repair are associated with male infertility (Gunes et al. 2015; Nagirnaja et al. 2018). However, the pathology of testicular degeneration of $\mathrm{Xpa}^{-/-}$mice remains unknown.

To maintain cellular homeostasis, autophagy is an intracellular degradation pathway, through which a portion of the cytoplasm is brought to lysosomes to be degraded and is stimulated in response to some stressful situations such as nutrient deprivation, oxidative stress, and DNA damage. Microtubule-associated protein 1 light chain 3 (LC3) is a ubiquitin-like protein and is initially synthesized as LC3-I (approximately $16-18 \mathrm{kDa}$ ), and then modified into the phosphatidylethanolamine (PE)-conjugated form, LC3-II on the surface of newly formed autophagosomes (Gomes et al. 2017; Yoshii and Mizushima 2017). Therefore, the level of LC3-II or the conversion of LC3-I to LC3-II are the most widely used peculiar markers associated with completed autopha- gosomes. XPA deficiency upregulates autophagy with mitochondrial dysfunction in XP group A patient's cells (Fang et al. 2014); XPA protein might affect mitochondrial maintenance and regulation of mitophagy (Manandhar et al. 2017). Besides, a study has also reported the induction of autophagy in NER-deficient mice (Marino et al. 2008). Based on the studies reporting impaired spermatogenesis and autophagy induction in the $X p a^{-t-}$ mice, we hypothesized that autophagy could be involved in the impaired spermatogenesis in $\mathrm{Xpa}^{-/-}$mice.

Here, to elucidate the pathology of testicular degeneration in Xpa deficiency, we investigated the testis of 3-month-old $\mathrm{Xpa}^{-/-}$mice compared to that of wt mice through immunohistochemistry and immunoblotting with anti-LC3 antibody. These findings suggest a novel role of autophagy in the testicular degeneration of the $\mathrm{Xpa}^{-/-}$mice.

\section{MATERIALS AND METHODS}

Mutant mice. The Xpa gene-knockout mice $\left(\mathrm{Xpa}^{-/-}\right.$ mice) were generated by the insertion of the neo gene into the exon 4 of mouse Xpa gene and had a chimeric genetic background of CBA/C57BL6/CD-1 (Nakane et al. 1995). The mice were kept under specific pathogen-free conditions, housed in a controlled environment at $20-26^{\circ} \mathrm{C}$, fed a CE-2 diet (Clea Japan Inc, Tokyo, Japan) and sterilized water ad libitum for an extended period. All animal experiments in this study were conducted following the guidelines for the institutional animal care and use committee (No.18-Y-13) and the safety committee for genetic recombination experiments (No.28-017) of Tottori University.

Tissue preparation. Three-month-old animals were deeply anesthetized under triple mixed anesthesia (medetomidine, midazolam and butorphanol). Testes were fixed overnight in Bouin's solution and then transferred to $70 \%$ ethanol. Mice were transcardially perfused with $4 \%$ paraformaldehyde (PFA) in $0.1 \mathrm{M}$ phosphate buffered saline (PBS) for immunohistochemistry. These organs were embedded in paraffin. Paraffin sections $3-\mu \mathrm{m}$-thick were prepared and stained with periodic acid-Schiff and hematoxylin $(\mathrm{PAS} / \mathrm{H})$. Cover-slipped sections were viewed, and the images were captured using a Nikon Eclipse E600 microscope (Nikon, Tokyo, Japan). To check the number of seminiferous tubules with large vacuoles (loss of several to many cells) (Fig. 1B, inset lower square), we observed 250 seminiferous tubules of each testis (7 control mice and $7 \mathrm{Xpa}^{-/-}$mice). 
Data on all pathological and immunohistochemical changes were obtained from at least three mice. For the immunoblotting of mouse testis, freshly removed mouse testes were weighed and frozen with liquid nitrogen and stored at $-80^{\circ} \mathrm{C}$ until the further experiment.

Protein extraction and immunoblotting. All the procedures were carried out at $4^{\circ} \mathrm{C}$. Tissues were lysed by sonication in $10 \mathrm{mM}$ Tris- $\mathrm{HCl}(\mathrm{pH} 7.4), 150 \mathrm{mM}$ $\mathrm{NaCl}, 1 \mathrm{mM}$ EDTA, $1 \mathrm{mM}$ EGTA, 1\% Triton X-100 and a protease inhibitor cocktail (Roche Diagnostics, Indianapolis, IN). The supernatants were centrifuged at $100,000 \times g$ for $30 \mathrm{~min}$, and the insoluble pellets were suspended in the same extraction buffer. Protein concentrations were determined using the Protein Assay Rapid Kit (WAKO, Tokyo, Japan). Immunoblotting was performed with rabbit polyclonal anti-LC3 Ab (MBL, Nagoya, Japan), and polyclonal anti-tubulin Ab (Santa Cruz Biotech, Santa Cruz, CA). Immuno-positive signals were visualized by ECL plus reagent (GE Healthcare, Buckinghamshire, UK), and images were obtained using LAS-4000 image analyzer (Fujifilm, Tokyo, Japan) (Takai et al. 2013).

Immunohistochemistry for LC3. LC3 immunohistochemistry was done with anti-LC3 Ab (microtubule-associated protein 1 light chain 3 ; MBL) at a dilution of $1 / 500$ by antigen retrieval, Immunosaver (Nisshin EM, Tokyo, Japan). The Histofine SABPO(R) Kit (Nichirei, Tokyo, Japan) was used for visualization of primary antibody binding. Sections were counterstained with hematoxylin.

Statistical analysis. All experimental data are reported as mean, and the error bars represent the standard deviation (SD). Statistical analysis was performed using the Unpaired Student's $t$-test. All the statistical analyses were performed using the KaleidaGraph v. 4.1.0 (Synergy software, Inc.).

\section{RESULTS}

Vacuolar formation in the testis of 3-month-old $\mathrm{Xpa}^{-1-}$ mice

Careful examination of the testis of $\mathrm{Xpa}^{-/-}$mice revealed disorganization of a substantial portion of the tubule structure with large vacuoles (Fig. 1B, inset, lower square) and small vacuoles (Fig. 1B, inset, upper square) in the testis of 3-month-old $\mathrm{Xpa}^{-/}$ mice, wherein the $\mathrm{Xpa}^{+/+}$mice had normal seminiferous tubules (Fig. 1A and B). The vacuoles were found to be distributed from the middle to the basal part of the seminiferous epithelium. Histological analysis revealed that the testis of $\mathrm{Xpa}^{-/-}$mice had a lot of seminiferous tubules with large vacuoles, while the testicular seminiferous tubules of wt mice had no large vacuoles (Fig. 1C). Small vacuoles were observed in the $\mathrm{Xpa}^{-/}$and $\mathrm{Xpa}^{+/+}$mice. The formation of large vacuoles in the testis of 3-month-old $\mathrm{Xpa}^{-/-}$mice indicated an early pathological change of impaired spermatogenesis.

Increased number of degenerating cells with LC3 signals in the testis of 3-month-old $\mathrm{Xpa}^{-/-}$mice

The immunohistochemistry analysis using anti-LC3 antibodies revealed the relationship between vacuole formation and the distribution of autophagy induction in the testis of $\mathrm{Xpa}^{-/-}$mice. The number of punctate LC3 structures (empty arrow head) detected in wt mice (Fig. 2A) were lower than those in the $\mathrm{Xpa}^{-/-}$mice testis (Fig. 2B and C). Besides, degenerating cells with higher signals of LC3 corresponding to the vacuole position were observed (Fig. 2B and $\mathrm{C}$, black arrow), indicating increased autophagy. Judging from the location of the vacuoles, these degenerating cells with signals of LC3 on the whole cells may be the spermatogonia or primary spermatocytes. Furthermore, the measurement of body weight revealed that the caloric intake was normal in $\mathrm{Xpa}^{-/-}$mice, thereby excluding malnutrition as a cause for the observed autophagy induction in $\mathrm{Xpa}^{-/-}$ mice. The results supported the assumption that vacuolar formation with reduction of testis weight might be due to autophagy induction in the testis of $\mathrm{Xpa}^{-/}$mice.

\section{Autophagy induction in the testis of 3-month-old} $\mathrm{Xpa}^{-/-}$mice

To confirm the autophagy induction in the testis of 3-month-old $\mathrm{Xpa}^{---}$mice, we evaluated the autophagic status by immunoblot analysis using anti-LC3 antibodies. We detected the increased levels of LC3-II recruited to autophagosomal membranes only in the testis of 3-month-old $\mathrm{Xpa}^{-/-}$mice, whereas there was no LC3-II band for the testis of wt mice.

In contrast to LC3-II, cytosolic LC3-I band was unchanged both $\mathrm{Xpa}^{--}$mice and wt mice (Fig. 3A). The autophagic activity in the testis from 3-monthold $\mathrm{Xpa}^{-/-}$mice was significantly higher than that observed in wt mice, as assessed by the increased LC3-II/LC3-I ratio in $\mathrm{Xpa}^{-/}$mice tissues (Unpaired Student's $t$-test: $P<0.05$ ) (Fig. 3B). The results supported the immunohistochemical results of LC3 (Fig. 2), indicating that the induction of autophagy 

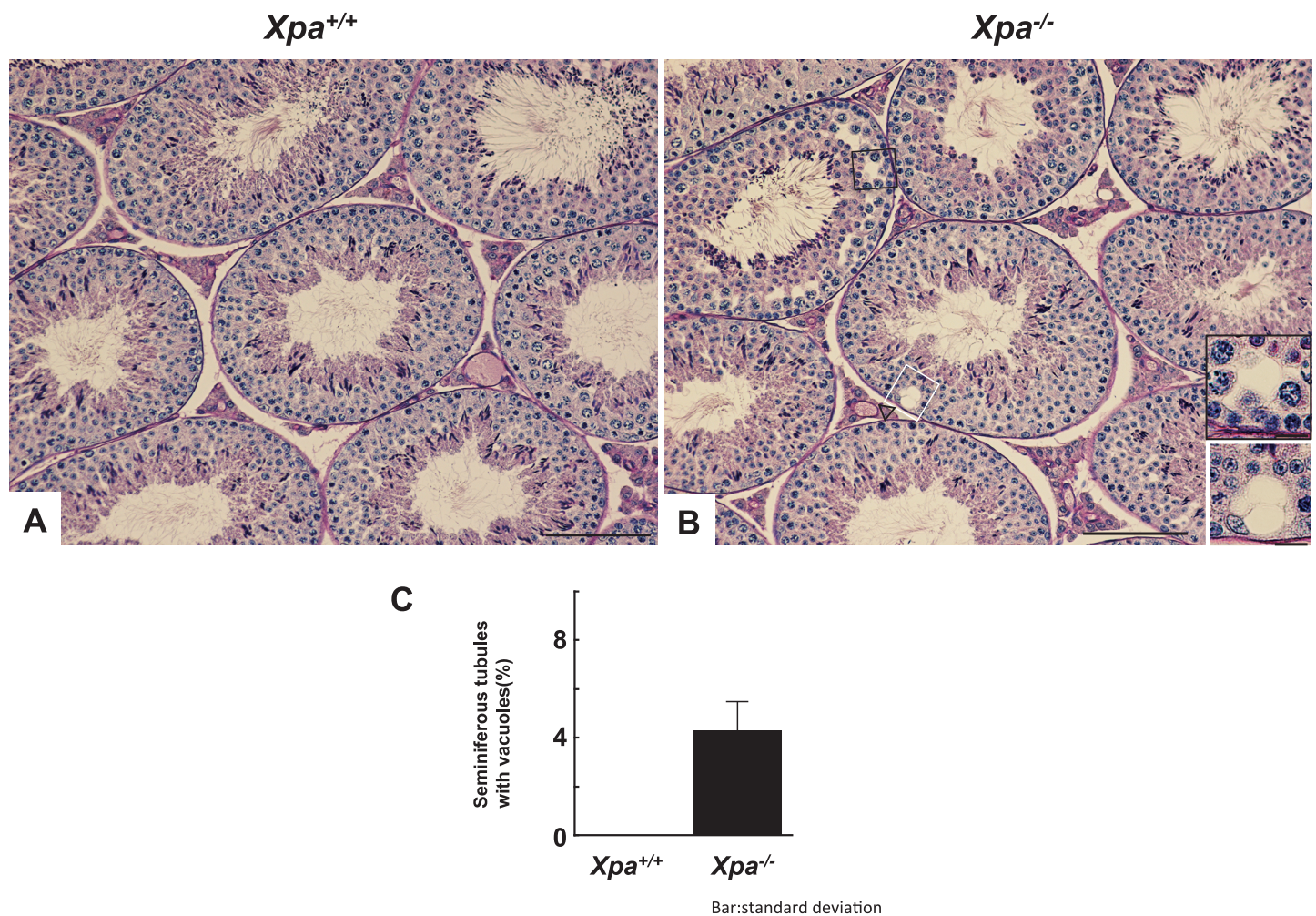

Fig. 1 View of seminiferous tubules with vacuoles in the testis of 3-month-old wt $\left(\mathrm{Xpa}^{+/+}\right)$and $X p a^{-/-}$mice. (A) The testis of 3-months-old $\mathrm{Xpa}^{+/+}$mice. (B) The testis of 3-months-old $\mathrm{Xpa}^{-/-}$mice. Microscopic view of seminiferous tubules; the $(\triangle)$ empty arrowhead indicates a large vacuole formed by the loss of several to many cells. The insets show higher-magnification images of the area captured by the squares (lower square: large vacuole, upper square: small vacuole). Bars: $100 \mu \mathrm{m}$ (A, B), $25 \mu \mathrm{m}$ (inset). (C) Percentage of the number of seminiferous tubules with vacuoles observed in the testis of 3-month-old wt $\left(\mathrm{Xpa}^{+/+}\right)$and $\mathrm{Xpa}^{-/-}$mice. The error bar represents the standard deviation (SD). Periodic acid-Schiff and hematoxylin $(\mathrm{PAS} / \mathrm{H})$ stain.

\section{$\mathrm{Xpa}^{+/+}$}

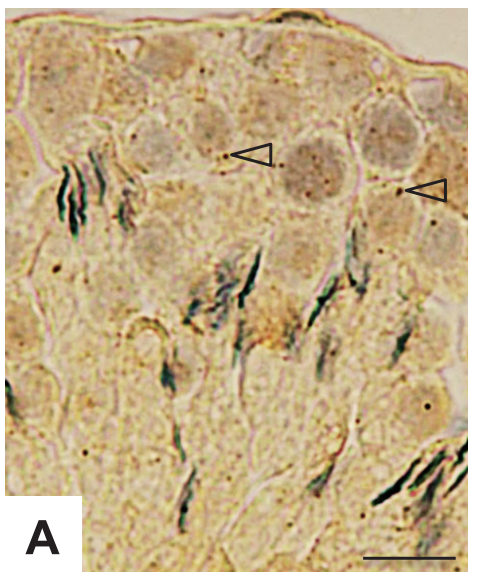

$X p a^{-/-}$
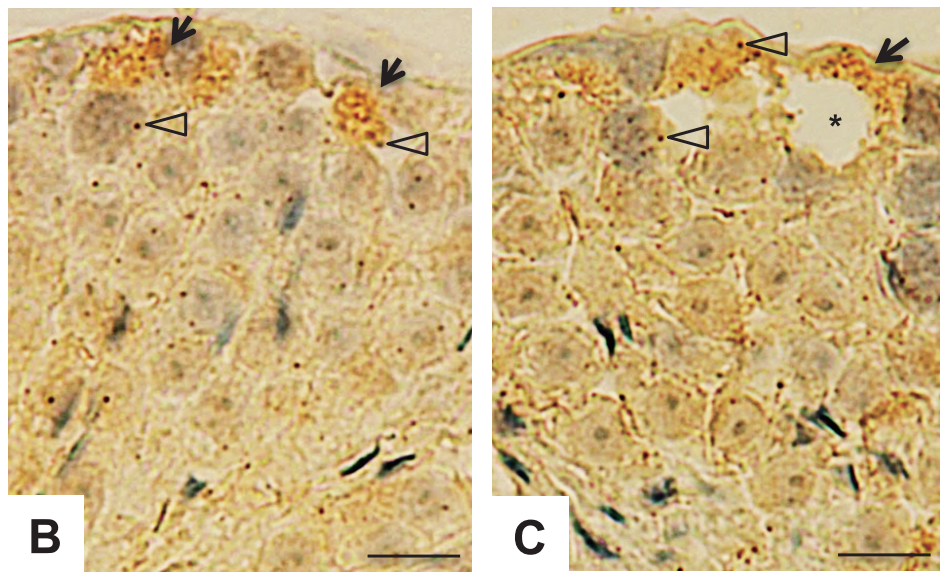

* : large vacuole

Fig. 2 Level of LC3 signals in the testis of 3-month-old wt and $X p a^{-/-}$mice. Empty arrow heads indicate LC3 signal. (A) Testis of $\mathrm{Xpa}^{+/+}$mice. (B, C) Testis of $\mathrm{Xpa}^{-/-}$mice with testicular cells having signals of LC3. Arrows of (B) represent the basal cells with LC3 signals and arrows of (C) represent the cells with LC3 signals corresponding to vacuoles of the seminiferous epithelium in $\mathrm{Xpa}^{-/-}$mice. The bar corresponds to $10 \mu \mathrm{m}$. Asterisk: large vacuole 
A

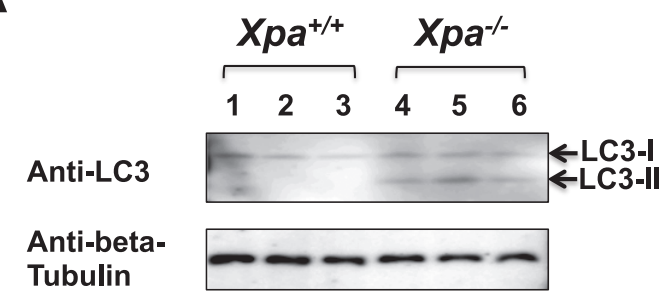

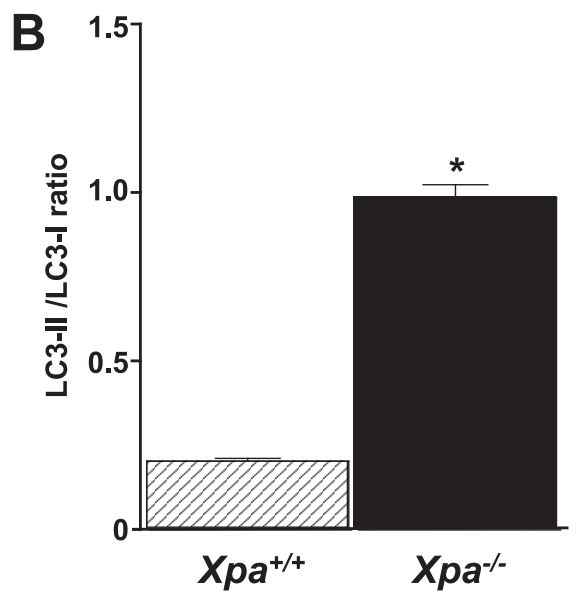

*Unpaired Student's $t$-test $(P<0.05)$

Fig. 3 Anti-LC3 immunoblotting in lysates from the testis of 3-month-old $X_{p a}{ }^{-/-}$mice. (A) Immunobloting using anti-LC3 antibody and anti-beta-tubulin antibody. Lane 1-3: LC3-I (first line), LC3-II (second line), and tubulin (lower panel) levels in the testis of $\mathrm{Xpa}^{+/+}$mice; Lane 4-6: LC3-I (first line), LC3-II (second line), and tubulin (lower panel) levels in the testis of $\mathrm{Xpa}^{-/-}$mice. (B) The LC3-II / LC3-I ratio in the testis of 3-months-old $\mathrm{Xpa}^{+/+}$and $\mathrm{Xpa}^{-/-}$mice. * The means were significant at $P<0.05$ (Unpaired Student's $t$-test). The error bars represent the standard deviation (SD).

with loss of testicular cells could be a specific alteration caused by Xpa deficiency.

\section{DISCUSSION}

The present study was carried out to understand the pathology underlying the impaired spermatogenesis in 3-month-old Xpa-deficient mice. The histochemical and immunoblot analyses revealed the presence of a higher number of large vacuoles with induced autophagy and increased levels of LC3-II/LC3-I ratio, important biomarkers of autophagy as compared to that in the wt mice. Here, we discuss the possible mechanistic explanations for these observations.

\section{Nucleotide excision repair (NER) deficiency and au- tophagy}

There are some reports that NER deficiency is associated with autophagy. One study has reported that $X P A$ deficiency upregulates autophagy, leading to mitochondrial and mitophagy dysfunction in the cells of XP group A patients (Fang et al. 2014). Another study has also reported the induction of autophagy in premature aging NER-deficient mice (Marino et al. 2008). Moreover, the $\mathrm{Xpa}^{-/-}$mice exhibited aging phenotypes such as impairment of spermatogenesis and higher incidence of spontaneous tumorigenesis (Nakane et al. 2008). Although we obtained similar results of induced autophagy in the testis compared to that in control through immunohistochemistry and immunoblotting assays in the liver of 3-month old
$\mathrm{Xpa}^{-/-}$mice, organ weight of the liver was not reduced (data not shown). Even if the same NER is deficient, the biological reaction may differ depending on the tissue. It can be concluded that NER deficiency in humans and mice induce autophagy.

Mechanisms of testicular abnormalities in $\mathrm{Xpa}^{-/-}$mice In seminiferous tubules of 3-month-old $\mathrm{Xpa}^{-/-}$mice, we observed many large vacuoles and degenerating cells with a higher level of LC3 signals. A study involving the autophagy-related gene 7 (Atg7)knockout infertile male mice reported the similar appearance of many large vacuoles in the seminiferous tubules which might originate from the dead germ cells compared to that in control mice (Wang et al. 2014). The appearance of large vacuoles can be considered as an early pathological change in the testis of 3-month-old $\mathrm{Xpa}^{--/}$mice. In our study, we detected a higher accumulation of cyclopurine in the brain, kidney, liver, and testis of 1-, 3-, 6-, 24-monthold $\mathrm{Xpa}^{-/-}$mice as compared to that in the wt mice in age-dependent manner. This suggested that agedependent accumulation of endogenous cyclopurine in the brain may be critical for neurological abnormalities of XP patients. Cyclopurine lesions appeared to accumulate in DNAs of the testis from 3-monthold $\mathrm{Xpa}^{-/}$mice (Mori et al. 2019). Therefore, the formation of large vacuoles in the testis of 3-monthold $\mathrm{Xpa}^{-/-}$mice could be attributed to the fact that the germ cells grow and differentiate continuously, and cyclopurine might be a stable block to tran- 
scription and replication in rapidly proliferating cells, thus inducing autophagy or cell death of the affected testicular cells leading to the formation of large vacuoles.

\section{Male infertility and decreased expression of XPA}

It has been shown that XPA deficiency is associated with male infertility as evident from the study carried out in humans, which showed a decreased expression of XPA in 143 of the 620 infertile men with a unique polymorphism in the promoter region of $X P A$ gene and also sperm DNA damages (Gu et al. 2010). Singh et al. (2019) reported that some azoospermic infertile patients with hypospermatogenesis and maturation arrest showed the low expression of $X P A$. Thus, decreased expression of $X P A$ in testis may be associated with male infertility.

We acknowledge that there are several limitations to this study. Firstly, we do not detect the status of autophagy such as autophagy flux, indicator of autophagic activity in vivo. Secondly, we do not reveal the cause and the cell type of induced autophagy in the $\mathrm{Xpa}^{-/-}$testis. To overcome these limits, we will examine the autophagy flux and the stage specific germ cell marker on degenerating cells and monitor DNA lesion levels in testicular cells of $\mathrm{Xpa}^{-/-}$mice. In summary, we propose autophagy induction in the testis of 3-month-old $\mathrm{Xpa}^{-/-}$mice as the underlying mechanism for impaired spermatogenesis. The $\mathrm{Xpa}^{-{ }^{-}}$ mice could be a valuable animal model to study pathological processes that affect human XP patients and male infertile patients with decreased expression of $X P A$. We believe that the present findings provide new insight into the mechanism of male infertility and will contribute to therapy for male infertility.

\section{CONFLICT OF INTERESTS}

The authors declare that there are no conflicts of interest.

\section{Acknowledgments}

The authors thank Dr. Kiyoji Tanaka for Xpa mice. We thank Dr. Philip J. Brooks (NIH) for critical reading of the manuscript and helpful discussions. This work was supported by a Grant-in-Aid for Scientific Research from the Ministry of Education, Culture, Sports, Science and Technology of Japan (JSPS KAKENHI Grant Numbers: JP19K09671, JP26462460, JP23592379 to H.N.). We thank Mr. Toshio Kameie for his excellent technical support. We would like to thank Editage (www.editage.com) for English language editing.

\section{REFERENCES}

Brooks PJ (2017) The cyclopurine deoxynucleosides: DNA repair, biological effects, mechanistic insights, and unanswered questions. Free Radic Biol Med 107, 90-100.

DeSanctis C and Cacchione A (1932) L'idiozia xerodermica. Riv Sper Freniatr 56, 269-292.

Fang EF, Scheibye-Knudsen M, Brace LE, Kassahun H, Sengupta $\mathrm{T}$, et al. (2014) Defective mitophagy in XPA via PARP-1 hyperactivation and $\mathrm{NAD}(+) / \mathrm{SIRT} 1$ reduction. Cell 157, 882-896.

Friedberg EC, Walker GC, Siede W, Wood RD, Schultz RA, et al. (2006) DNA Repair and Mutagenesis, ASM Press, Washington, DC.

Gomes LR, Menck CFM and Leandro GS (2017) Autophagy roles in the modulation of DNA repair pathways. Int $J \mathrm{Mol}$ Sci 18, 2351.

Gu A, Ji G, Zhu P, Zhou Y, Fu G, et al. (2010) Nucleotide excision repair polymorphisms, polycyclic aromatic hydrocarbon exposure, and their effects on sperm deoxyribonucleic acid damage and male factor infertility. Fertil Steril 94, 2620 2625.

Gunes S, Al-Sadaan M and Agarwal A (2015) Spermatogenesis, DNA damage and DNA repair mechanisms in male infertility. Reprod Biomed Online 31, 309-319.

Manandhar M, Lowery MG, Boulware KS, Lin KH, Lu Y, et al. (2017) Transcriptional consequences of XPA disruption in human cell lines. DNA Repair (Amst) 57, 76-90.

Marino G, Ugalde AP, Salvador-Montoliu N, Varela I, Quiros PM, et al. (2008) Premature aging in mice activates a systemic metabolic response involving autophagy induction. Hum Mol Genet 17, 2196-2211.

Mori T, Nakane H, Iwamoto T, Krokidis MG, Chatgilialoglu C, et al. (2019) High levels of oxidatively generated DNA damage $8,5^{\prime}$ '-cyclo-2'-deoxyadenosine accumulate in the brain tissues of xeroderma pigmentosum group A gene-knockout mice. DNA Repair (Amst) 80, 52-58.

Nagirnaja L, Aston KI and Conrad DF (2018) Genetic intersection of male infertility and cancer. Fertil Steril 109, 20-26.

Nakane H, Takeuchi S, Yuba S, Saijo M, Nakatsu Y, et al. (1995) High incidence of ultraviolet-B-or chemical-carcinogen-induced skin tumors in mice lacking the xeroderma pigmentosum group A gene. Nature 377, 165-168.

Nakane H, Hirota S, Brooks PJ, Nakabeppu Y, Nakatsu Y, et al. (2008) Impaired spermatogenesis, and elevated spontaneous tumorigenesis in xeroderma pigmentosum group A gene (Xpa)-deficient mice. DNA Repair (Amst) 7, 1938-1950.

Singh V, Jaiswal D, Singh K, Trivedi S, Agrawal NK, et al. (2019) Azoospermic infertility is associated with altered expression of DNA repair genes. DNA Repair (Amst) 75, 39-47.

Sugasawa K (2016) Molecular mechanisms of DNA damage recognition for mammalian nucleotide excision repair. DNA Repair (Amst) 44, 110-117.

Takai T, Higaki K, Aguilar-Moncayo M, Mena-Barragan T, Hirano $\mathrm{Y}$, et al. (2013) A bicyclic 1-deoxygalactonojirimycin derivative as a novel pharmacological chaperone for GM1 gangliosidosis. Mol Ther 21, 526-532.

Wang H, Wan H, Li X, Liu W, Chen Q, et al. (2014) Atg7 is required for acrosome biogenesis during spermatogenesis in mice. Cell Res 24, 852-869.

Yoshii SR and Mizushima N (2017) Monitoring and measuring autophagy. Int J Mol Sci 18, 1865. 